\title{
Suppressive effects of phosphorylated ascorbate on ultraviolet B radiation-induced DNA damage and differential expression of the wild-type and mutated p53 tumor-suppressor gene in keratinocytes
}

\author{
MIHO SUGIMOTO $^{1}$, YASUKAZU SAITOH ${ }^{1}$, MASAMITSU ICHIHASHI ${ }^{2}$ and NOBUHIKO MIWA ${ }^{1}$ \\ ${ }^{1}$ Laboratory of Cell-Death Control Biotechnology, Faculty of Life and Environmental Sciences, \\ Prefectural University of Hiroshima, Shobara, Hiroshima 727-0023; 2 Department of Dermatology, \\ Kobe University School of Medicine, Chuo, Kobe 650, Japan
}

Received May 14, 2009; Accepted July 6, 2009

DOI: 10.3892/mmr_00000192

\begin{abstract}
Ultraviolet B radiation (UVB)-induced DNA damage such as cyclobutane pyrimidine dimers (CPDs) and (6-4) photoproducts (6-4PP) in mouse epidermal keratinocytes (Pam212) was suppressed by pre-irradiation administration of two autooxidation-resistant vitamin $\mathrm{C}$ derivatives, ascorbic acid (Asc)-2-O-phosphate (Asc2P) and dehydro-Asc, but not by Asc or Asc-2-O- $\alpha$-glucoside (Asc2G). According to Western blot analysis of the UVB-irradiated keratinocytes, expression of the tumor suppressor gene p53 was immediately enhanced from low pre-irradiation levels to the nearly maximum level at $2 \mathrm{~h}$ post-irradiation. In contrast, the p53-antagonizing oncogene mdm2 was scarcely expressed at $0-1 \mathrm{~h}$ post-irradiation, but was drastically enhanced at 2-3 h post-irradiation, eventually paralleling p53 expression. This suggests that the period for the actual effective function of $\mathrm{p} 53$ proteins may be restricted to the initial 0-3 $\mathrm{h}$ after UVB irradiation. p53 expression reached the maximum at $3 \mathrm{~h}$ post-irradiation and remained unchanged until at least $6 \mathrm{~h}$, reaching its maximum at UVB doses of 20-100 mJ/ $\mathrm{cm}^{2}$ - nearly equal to the minimum erythema dose (MED) or to the doubled score for human skin. However, at 200-500 mJ/ $\mathrm{cm}^{2}$, p53 expression was markedly suppressed. This suggests a risk for potential photocarcinogenesis at doses greater than double the MED. UVB-induced p53 expression was notably suppressed by Asc2P or dehydro-Asc, but not by Asc. Nuclear
\end{abstract}

Correspondence to: Professor Nobuhiko Miwa, Laboratory of CellDeath Control Biotechnology, Faculty of Life and Environmental Sciences, Prefectural University of Hiroshima, Nanatsuka 562, Shobara, Hiroshima 727-0023, Japan

E-mail: miwa-nob@pu-hiroshima.ac.jp

*Contributed equally

Key words: ultraviolet, ascorbic acid-2-O-phosphate, p53, pyrimidine dimer, (6-4)photoproducts expression of the wild-type p53 gene (wt-p53) was markedly promoted at $6 \mathrm{~h}$ post-irradiation, and significantly suppressed by Asc, Asc2G or dehydro-Asc, as well as by Asc2P. In contrast, mutation-type p53 (mt-p53), which is known to interfere with wt-p53 function, was not markedly expressed in the nuclei and was explicitly suppressed by Asc2P but not by Asc, dehydro-Asc or Asc $2 \mathrm{G}$, which was not cytoprotective against UVB. Thus, compared to non-cytoprotective Asc or the other derivatives, Asc2P suppressed UVB-induced CPD and 6-4PP formation, resulting in both cytoprotective effects and the preferential suppression of mt-p53 over wt-p53.

\section{Introduction}

Skin undergoes oxidative stress attributed mainly to direct exposure to sunlight, and therefore needs to be protected against ultraviolet (UV) radiation injuries. A defensive role against external stress is played by the epidermis, which consists of keratinocytes and melanocytes, rather than by the dermis. Keratinocytes undergo repeated cell division and differentiation and cope with various stresses from the inside and outside of an organism. It is well known that active oxygen species (ROS) contribute to this oxidative damage; however, dermal ROS-scavenging ability is insufficient on its own, and it is thought that the antioxidation ability of keratinocytes needs to be enhanced.

UV radiation causes various types of injuries, including DNA strand cleavages (1-3) and pyrimidine base damage such as cyclobutane pyrimidine dimers (CPD) and (6-4)photoproducts (6-4PP) (4). These contribute to cell senescence and deterioration as well as to cellular multiplication and differentiation $(5,6)$. Repair of DNA damage is necessary for the restoration of complete cellular function when mutations and deletions occur in key genes.

Cells suffering from extensive irreparable UV-induced DNA damage should undergo apoptosis initiated by the translocation of $\mathrm{p} 53$ proteins to the nucleus and their accumulation in the genetic transcription loci of the target gene. The cell cycle is transiently arrested in the G1 phase by $\mathrm{p} 53$ proteins 
when DNA damage is sustained, and its resumption is delayed in order to stall for DNA repair time; in turn, cells failing to repair themselves undergo apoptosis in order to prevent carcinogenesis (7).

MDM2 proteins induced by p53 gene expression specifically bind to the transcriptional locus of the p53 gene and, as a result, play a role in the inhibition of the transcription activity of p53 and the decomposition of p53 proteins (8). Variations in $\mathrm{p} 53$ protein function are known to exert a malignant rather than a neutral effect $(9,10)$, since variant $\mathrm{p} 53$ acts in a dominant negative fashion to hinder the normal function of wild-type p53 (wt-p53) (11,12).

Furthermore, diverse types of ROS, which are harmful to the execution of cellular functions, are generated when cutaneous tissues are irradiated with UV rays (13). ROS is so labile that it injures protein lipids and DNA, which are biogenic components (14).

Therefore, in the present study, ascorbic acid (Asc) and its derivatives were examined in terms of DNA defense against UV lesions, since Asc scavenges ROS in the aqueous phase more rapidly than the numerous endogenous antioxidants in an organism (15). In addition, the differential expression of the wt-p53 and mt-p53 genes was examined in conjunction with the preventive effects of Asc derivatives in DNA lesions.

\section{Materials and methods}

Cell culture. Mouse epidermal keratinocytes (Pam212) were a generous gift from Dr T. Fujimoto (Kyoto University). Human skin fibroblasts (Fibro2Y cell line) were a generous gift from Dr M. Ichihashi (Kobe University). Cells were cultured in Dulbecco's modified Eagle's medium and minimum essential medium supplemented with $4 \mathrm{mM}$ L-glutamine, $10 \mathrm{U} / \mathrm{ml}$ penicillin, $5 \mu \mathrm{g} / \mathrm{ml}$ streptomycin and $10 \%$ fetal bovine serum (FBS) in a $5 \% \mathrm{CO}_{2}$ humidified atmosphere at $37^{\circ} \mathrm{C}$.

Ultraviolet irradiation. Cells grown to the logarithmic phase were seeded at densities of $0.25-2.0 \times 10^{4}$ cells $/ \mathrm{cm}^{2}$ in a $100-\mathrm{mm}$ dish or glass slide so as to proliferate to the subconfluent state. Subsequently, Asc (Sigma-Aldrich, Tokyo, Japan), Asc2-O-phosphate (Asc2P) (Showa Denko Inc., Tokyo, Japan), Asc-2-O- $\alpha$-glucoside (Asc2G) (Wako Pure Chemicals Co., Osaka, Japan) or dehydro-Asc (Sigma-Aldrich) was administered to the cells for 2 or $24 \mathrm{~h}$. After aspiration or rinsing with phosphate-buffered saline (PBS), cells were irradiated or not with a Spectronics UV trans-illuminator EBF-260 (maximal wavelength, $312 \mathrm{~nm}$; half-peak intensity range, 297-328 nm) under moistening conditions so as to prevent dryness. UV intensity was calibrated with an Ieda UV response meter VLX-312.

Slot blotting. Cells processed by each method were collected and washed twice in PBS. DNA was extracted with a DNA extraction kit (Wako), colorimetrically quantified with Hoechst 33258 (Wako) and restrictively digested with BamH1 (Gibco BRL, CA, USA). The extracted DNA was transferred to a membrane (Zeta-Probe Blotting Membrane; Bio-Rad, CA, USA) using a dot blotter (Sanplatec, Osaka, Japan). After washing with $2 \mathrm{X}$ saline sodium citrate, the membrane was blocked with $5 \%$ evaporated milk in TBS for $2 \mathrm{~h}$. Anti-CPD

\section{Pyrmidine Dimers (no additive)}

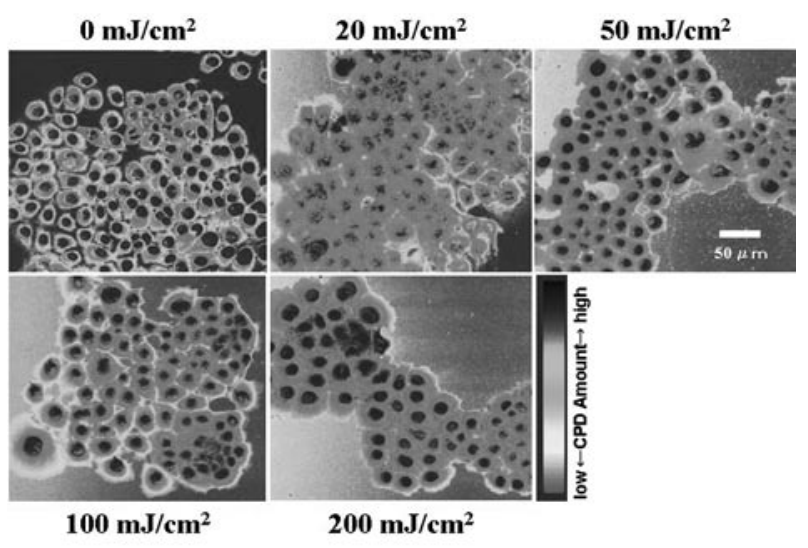

(6-4)Photoproducts (no additive)

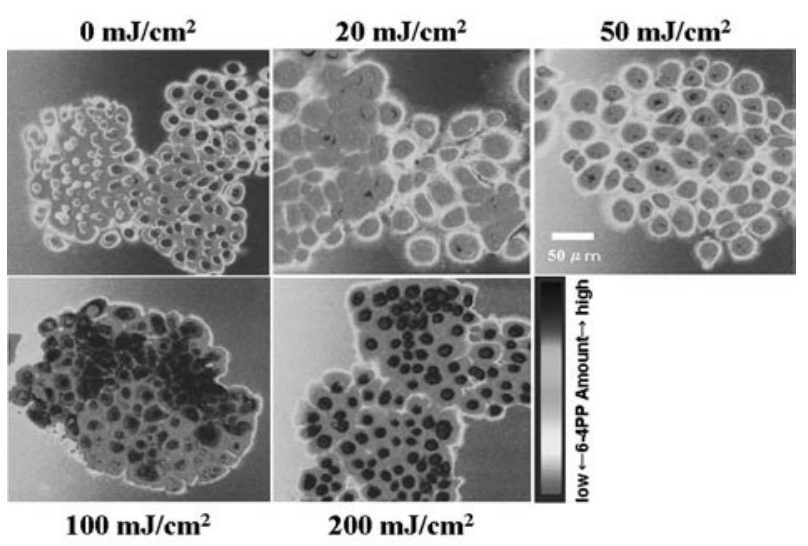

Figure 1. Dose-dependent formation of DNA damage, such as cyclobutane pyrimidine dimers (CPD) and (6-4)photoproducts (6-4PP), in mouse epidermal keratinocytes (Pam212) irradiated with graded doses of UVB. UVB-irradiated cells were immediately fixed and stained with antibody $(\mathrm{Ab})$ against $\mathrm{CPD}$ or 6-4PP as well as FITC-conjugated anti-IgG Ab, and subjected to fluorography. The resulting fluorescence intensity was processed to be artificially colorized with gradation from red to purple in the rainbow-color gauge in accordance with FITC-based fluorescence intensity.

and anti-6-4PP antibodies (a generous gift from Dr Nikaido, Kanazawa University) were incubated with the cells for $1 \mathrm{~h}$ at room temperature. After washing four times with TBS-T buffer (0.05\% Tween-20 in TBS), cells were incubated with horseradish peroxidase (HRP)-conjugated protein A (Amersham, $\mathrm{UK}$ ) for $45 \mathrm{~min}$ at room temperature, and then visualized by electrochemiluminescence (ECL) (Amersham). The density of the band was evaluated by NIH image software.

Western blot analysis. Cells processed by each method were harvested, treated with lysis solution (50 mM Tris- $\mathrm{HCl} \mathrm{pH} \mathrm{8.0,}$ $1 \mathrm{mM}$ EDTA, $150 \mathrm{mM}$ sodium chloride, $1 \mathrm{mM}$ DTT, $1 \%$ NP-40, $1 \mathrm{mM}$ PMSF, $10 \mu \mathrm{g} / \mathrm{ml}$ aprotinin, $10 \mu \mathrm{g} / \mathrm{ml}$ leupeptin) and frozen at $-80^{\circ} \mathrm{C}$. After thawing, the cells were ultrasonically crushed on ice for $5 \mathrm{sec}$ and separated by refrigerated centrifugation. The resulting supernatants were electrophoresed on an SDS-PAGE plate, and the collected protein was electrically transferred to a membrane (Millipore, MA, USA). The membrane was blocked with $5 \%$ evaporated milk in TBS for $2 \mathrm{~h}$, and then the cells were incubated with the primary antibodies 
(anti-p53, anti-MDM2; Santa Cruz, CA, USA) for $1 \mathrm{~h}$ at room temperature. After washing with TBS-T, cells were incubated with HRP-conjugated secondary antibody (Santa Cruz) for $1 \mathrm{~h}$ at room temperature, and then visualized by ECL.

Immunocytochemical staining of wild-type p53, mutated p53 or MDM2. Cells were seeded onto glass slides and treated by each processing method, then fixed by immersion in ice-cold acetone for $10 \mathrm{~min}$. Subsequently, the slides were washed four times with PBS and incubated with the primary antibodies [anti-wt-p53, mutation-type p53 (mt-p53), anti-MDM2] for $45 \mathrm{~min}$ at room temperature. After washing another four times with PBS, the slides were incubated with FITC-conjugated secondary antibody (Zymed Laboratories Inc., San Fransisco, CA, USA) for $35 \mathrm{~min}$. The reaction was terminated after washing four times with PBS, and was examined using a Bio-Rad confocal laser fluorescence scanning microscope.

Detection of cyclobutane pyrimidine dimers and (6-4)photoproducts. Cells processed by each method were seeded onto glass slides and fixed by immersion in ice-cold $70 \%$ ethanol for $15 \mathrm{~min}$, then frozen at $-20^{\circ} \mathrm{C}$. The slides were treated with $70 \mathrm{mM}$ sodium hydroxide for $2 \mathrm{~min}$ and washed three times with PBS, then treated with proteinase $\mathrm{K}$ for $15 \mathrm{~min}$ at room temperature. Slides were blocked with $10 \%$ FBS in PBS for 30 min and washed three times with PBS, then incubated with the primary antibodies (anti-CPD, 6-4PP) for $40 \mathrm{~min}$. After washing another three times with PBS, FITC-conjugated secondary antibody was added and reacted for $40 \mathrm{~min}$. The reaction was terminated and examined as described above.

\section{Results}

Dependence of UVB-induced DNA base injuries on ultraviolet doses. Pam 212 keratinocytes were irradiated with ultraviolet B rays (UVB) at the indicated doses, and the formation of DNA base injuries, such as CPD and 6-4PP, was detected by immunocytochemical staining using an antibody against CPD or 6-4PP as well as a FITC-conjugated anti-immunoglobulin antibody. CPD was markedly generated by UVB irradiation, even at doses as low as $20-50 \mathrm{~mJ} / \mathrm{cm}^{2}$, and increased in a dosedependent manner with UVB doses of up to $200 \mathrm{~mJ} / \mathrm{cm}^{2}$. 6-4PP was significantly generated by UVB irradiation at $20 \mathrm{~mJ} / \mathrm{cm}^{2}$, but marked generation occurred at $50-100 \mathrm{~mJ} / \mathrm{cm}^{2}$, a response less susceptible than that observed with CPD. 6-4PP was also increasingly generated in a manner dependent on the UVB dose, up to a dose of $200 \mathrm{~mJ} / \mathrm{cm}^{2}$ (Fig. 1).

Preventive effects of ascorbic acid derivatives on the formation of UVB-induced DNA base injuries. The inhibitory effects of Asc and its derivatives (dehydro-Asc, Asc2P and Asc2G) were examined in relation to the generation of CPD and 6-4PP. Pam 212 keratinocytes were treated with $200 \mu \mathrm{M}$ Asc or Asc derivates for $24 \mathrm{~h}$ before UV irradiation, and were then irradiated with UVB at $20 \mathrm{~mJ} / \mathrm{cm}^{2}$. The resulting CPD and 6-4PP damage was detected by slot blotting. CPD and 6-4PP damage was markedly inhibited by $200 \mu \mathrm{M}$ Asc $2 \mathrm{P}$ or dehydro-Asc. Asc2P was efficiently converted to Asc, as shown in our previous study (12). The inhibition rates for CPD formation were $20.7 \%$ for Asc, $14.1 \%$ for Asc2P, $13.2 \%$ for

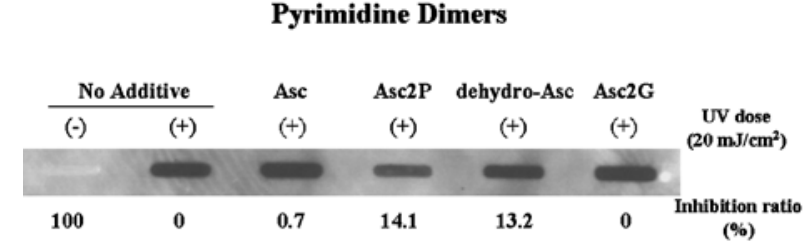

(6-4)Photoproducts

\begin{tabular}{|c|c|c|c|c|c|c|}
\hline \multicolumn{2}{|c|}{ No Additive } & Asc & Asc2P & dehydro-Asc & Asc2G & \multirow{3}{*}{$\begin{array}{c}\text { UV dose } \\
\left(20 \mathrm{~mJ} / \mathrm{cm}^{2}\right)\end{array}$} \\
\hline$(-)$ & $(+)$ & $(+)$ & $(+)$ & $(+)$ & $(+)$ & \\
\hline 60 & $=$ & & & & & \\
\hline 0 & 100 & 36.5 & 40.7 & 45.3 & 31.8 & $\begin{array}{l}\text { Inhibition ratio } \\
\text { (\%) }\end{array}$ \\
\hline
\end{tabular}

Figure 2. Correlation between DNA damage caused by UVB irradiation at $20 \mathrm{~mJ} /$ $\mathrm{cm}^{2}$ in Pam212 keratinocytes and cytoprotection by pre-irradiation administration for $24 \mathrm{~h}$ of $200 \mu \mathrm{M}$ Asc derivatives. The inhibitory effects of Asc derivatives on the formation of DNA damage, such as CPD and 6-4PP, were examined by slot blotting using antibodies against CPD or 6-4PP. The resulting chemiluminescence was detected and exhibited as a band on the slot.

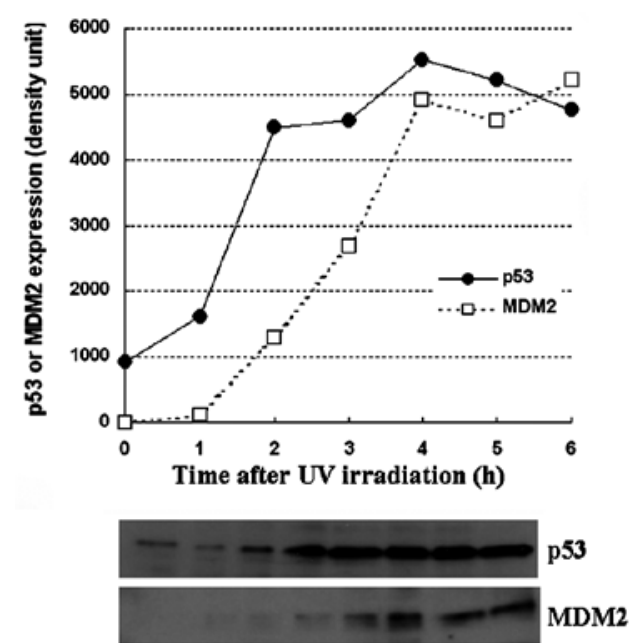

$(-)(+)(+)(+)(+)(+)(+)(+)$ UV irradiation \begin{tabular}{llllllll}
0 & 0 & 1 & 2 & 3 & 4 & 5 & 6 \\
\hline
\end{tabular}

Figure 3. Time course of the expression of the tumor suppressor gene p53 and the oncogene mdm2 after UVB irradiation of Pam 212 keratinocytes, as assessed by Western blot analysis and subsequent densitometric analysis.

dehydro-Asc and $0 \%$ for Asc2G. The inhibition rates for 6-4PP formation were $36.5 \%$ for Asc, $40.7 \%$ for Asc2P, $45.3 \%$ for dehydro-Asc and $31.8 \%$ for Asc2G (Fig. 2). In addition, $65 \mu \mathrm{M}$ Asc2P was administered at $2 \mathrm{~h}$ prior to UVB irradiation, and CPD and 6-4PP damage was inhibited even with the administration of UVB doses as high as $300 \mathrm{~mJ} / \mathrm{cm}^{2}$ (data not shown).

Effects of ascorbic acid derivatives on UVB-induced expression of p53 and MDM2. Expression of p53 and MDM2 after irradiation with $20 \mathrm{~mJ} / \mathrm{cm}^{2} \mathrm{UVB}$ was detected by Western blot analysis. Increases in the expression of p53 were observed $1 \mathrm{~h}$ after UVB irradiation and peaked 2 or $3 \mathrm{~h}$ later. Thereafter, p53 expression was maintained at an almost constant level, at least 

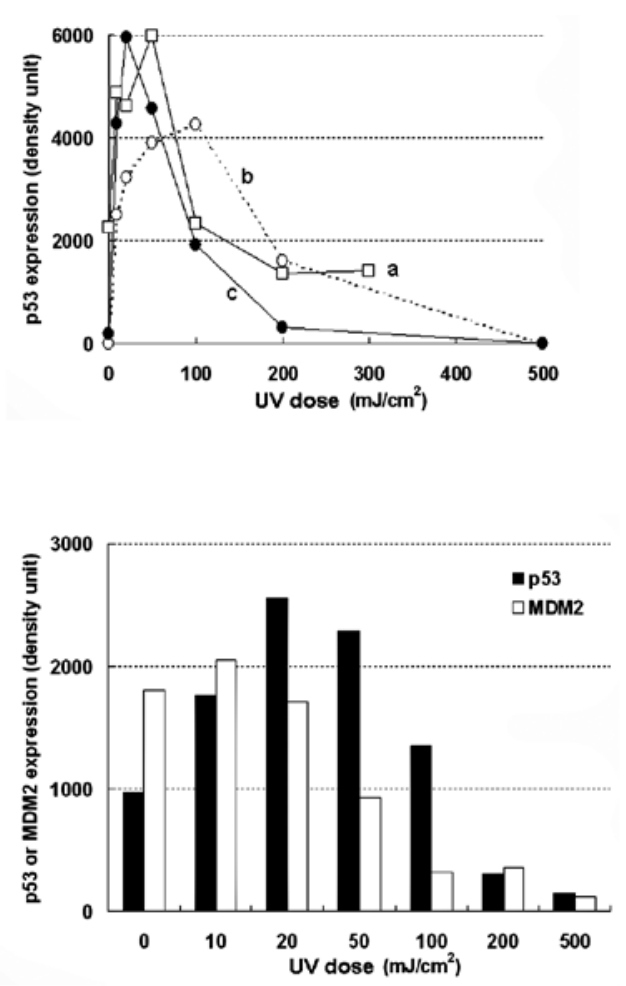

Figure 4. Dose-dependent p53 gene expression at $6 \mathrm{~h}$ (a) or $10 \mathrm{~h}$ (b and c), after the UVB irradiation of Pam212 keratinocytes, assessed by Western blot analysis and subsequent densitometric analysis.
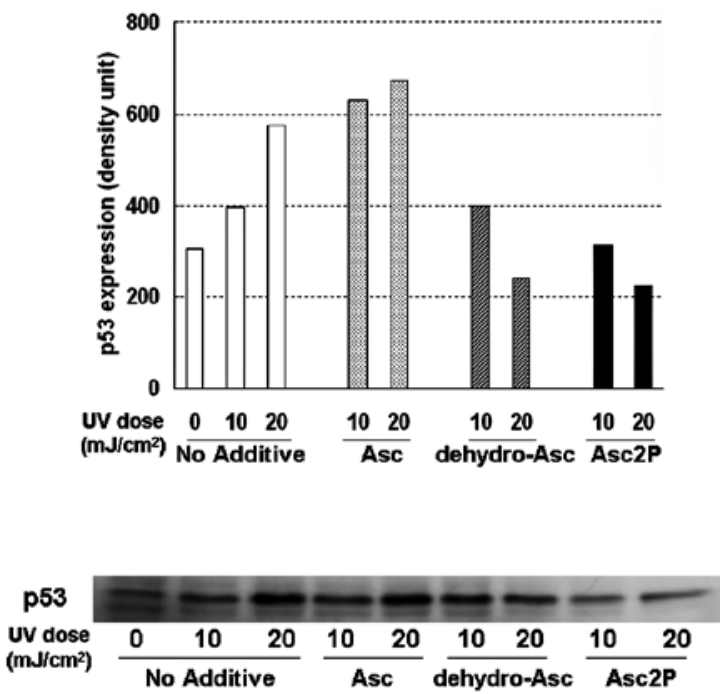

Figure 5. Inhibitory effects of $65 \mu \mathrm{M}$ Asc derivatives on UVB-promoted expression of the p53 gene at $6 \mathrm{~h}$ post-irradiation of Pam212 keratinocytes with UVB at the indicated doses, assessed by Western blot analysis using an anti-p53 protein antibody.

after $6 \mathrm{~h}$. In contrast, expression of MDM2 was delayed by $2 \mathrm{~h}$ compared to $\mathrm{p} 53$ expression, then increased and peaked 4 or 5 h later (Fig. 3). Expression of p53 and MDM2 was correlated with the UVB dose, confirmed by Western blot analysis.

At 6 or $10 \mathrm{~h}$ after UVB irradiation, p53 expression peaked at doses of $20-100 \mathrm{~mJ} / \mathrm{cm}^{2}$, and was suppressed at higher doses. In contrast, MDM2 expression peaked at a dose as low as $10 \mathrm{~mJ} / \mathrm{cm}^{2}$ (Fig. 4). After the administration of $65 \mu \mathrm{M}$ Asc
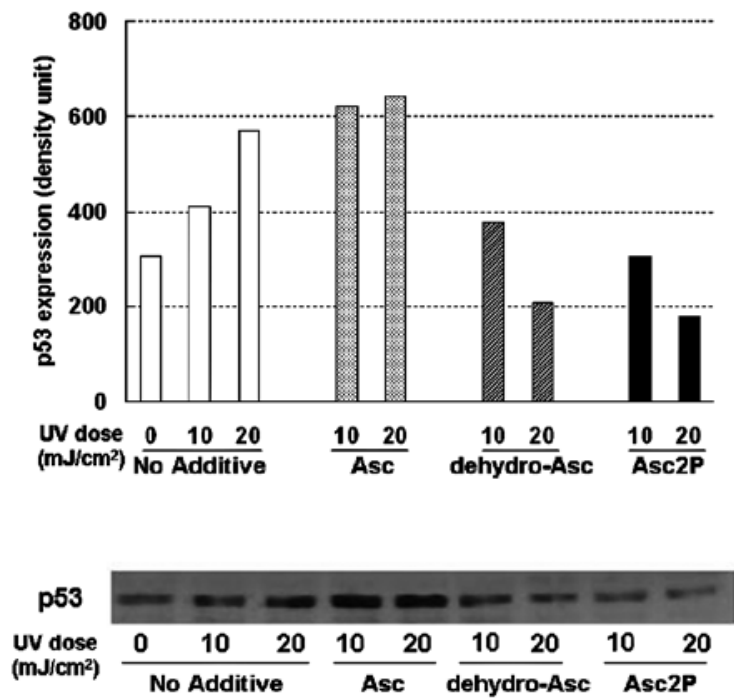

Figure 6. Inhibitory effects of $65 \mu \mathrm{M}$ Asc derivatives on UVB-promoted expression of the p53 gene at $6 \mathrm{~h}$ post-irradiation with UVB of Fibro2Y fibroblasts at the indicated doses, assessed by Western blot analysis using an anti-p53 protein antibody.

or of each Asc derivative at $2 \mathrm{~h}$ prior to UVB irradiation, cells were irradiated with 10 or $20 \mathrm{~mJ} / \mathrm{cm}^{2}$ UVB. p53 expression at $6 \mathrm{~h}$ after UV irradiation was quantified by Western blotting. UVB-induced expression of $\mathrm{p} 53$ was inhibited by Asc2P and dehydo-Asc, which were shown to suppress CPD and 6-4PP damage, but not by Asc. This result was similar to that observed in human and mouse keratinocytes (Figs. 5 and 6).

Ultraviolet B-induced differential expression of wild-type and mutated $p 53$ and the effects of ascorbic acid derivatives. Anti-wt-p53 and anti-mt-p53 antibodies were used for immunocytochemical staining to detect the association between DNA damage and the wild- and mutation-types of p53 that developed concurrently with UV-induced DNA lesions and their Asc-derivative-dependent suppression. At $6 \mathrm{~h}$ after $20 \mathrm{~mJ} /$ $\mathrm{cm}^{2}$ of UVB, wt-p53 proteins were strongly expressed within the nucleus, in contrast to the weaker expresison of mt-p53 proteins in the cytoplasm around the nucleus. Administration of Asc2P at $2 \mathrm{~h}$ prior to UVB irradiation moderately inhibited the expression of wt-p53 proteins and simultaneously markedly inhibited mt-p53 protein expression to the level observed before the abrupt increase. In contrast, administration of other Asc derivatives at $2 \mathrm{~h}$ prior to UV irradiation enhanced the expression of mt-p53 proteins, although wt-p53 was inhibited (Fig. 7). MDM2 expression was not induced by dehydro-Asc or Asc2G administration (data not shown).

\section{Discussion}

UV irradiation causes diverse types of DNA damage, in particular CPDs and 6-4PPs. These are formed when two adjacent pyrimidine bases are joined by a cross-link, mainly by means of the DNA-directed action of UV radiation. In the present study, we showed that CPD and 6-4PP damage was generated in a dose-dependent manner by UVB irradiation. This damage was prevented by Asc2P and dehydro-Asc, but 
Wild-type $\mathbf{p 3}$

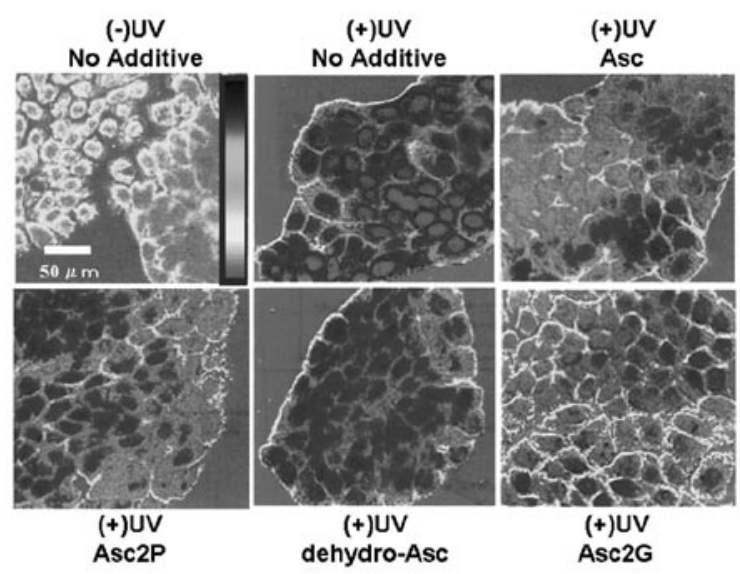

Mutated p53

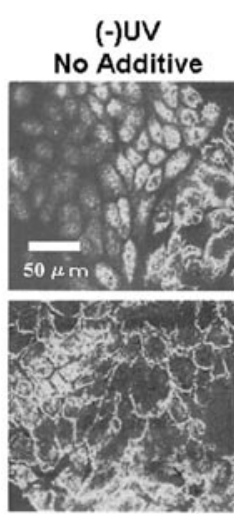

(+)UV

Asc2P

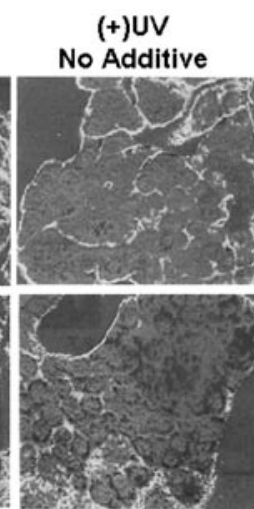

(+)UV

dehydro-Asc

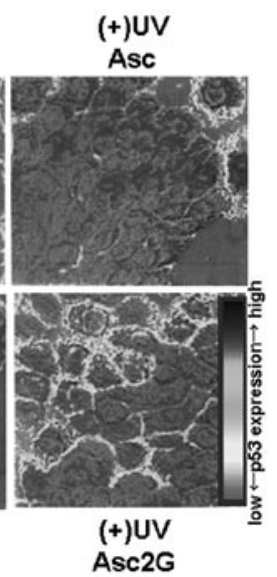

Asc2G
Figure 7. In situ detection of expression of wild-type (wt)-p53 or mutationtype (mt)-p53 in Pam212 keratinocytes, as shown by immunocytochemical staining using antibodies against wt-p53 or mt-p53 protein, followed by pseudo-color processing. Cells were previously treated with or without $65 \mu \mathrm{M}$ Asc derivatives, and then irradiated with $20 \mathrm{~mJ} / \mathrm{cm}^{2} \mathrm{UVB}$. After $6 \mathrm{~h}$, the cells were fixed and underwent immunocytochemical staining.

not by Asc or Asc2G. The results suggest that Asc2P and dehydro-Asc inhibit DNA damage caused by radiation due to a DNA base-directed energy-transfer action of the UVB rays, or that Asc2P and dehydro-Asc exert an antioxidant effect partially responsible for systemic assistance to cellular restorative and repair ability against UVB-derived wide-range injuries. On the other hand, UVB-induced DNA damage may be attributed, at least in part, to an oxidative effect such as the UV-induced generation of ROS, including singlet oxygen, which is considered to be scavenged by the oxidative decomposition-resistant vitamin $\mathrm{C}$ derivatives Asc2P or dehydro-Asc. Alternatively, Asc2P and dehydro-Asc may contribute to complete-cellular holonic effects responsible for DNA repair-supporting cellular stamina, attributed to Asc2P-dehydro-Asc-promoted antioxidant ability.

Asc2P is esterolytically converted to Asc by means of diverse ecto-enzyme types of phosphatases and taken up easily and continuously into the intracellular sphere via ascorbate transporters including SVCT-1 and -2 , followed by the intracellular accumulation of higher concentrations of Asc, as demonstrated in our previous studies (16-18). Asc and other Asc derivatives do not exert this action in mouse epidermal keratinocytes in the presence of $10 \%$ FBS. In this respect, exogenously administered Asc is so labile as to be oxidatively decomposed to diketogulonic acid and diverse reductones via dehydro-Asc, whereas other Asc derivatives, such as Asc2G and Asc-2-O-sulphate, scarcely undergo Asc conversion due to extreme stability or low susceptibility to $\alpha$-glucosidases or sulphatases lacking human or mouse skin cells and tissues upon cultivation with FBS $(19,20)$. In our previous study (21), we demonstrated that Asc2P prevents the UVB-induced fragmentation of DNA and apoptotic cell death through its conversion of Asc and the enrichment and accumulation of intracellular Asc. It has been suggested that intracellular Asc inhibits ROS generated by UV irradiation (22) and prevents DNA damage through the normal maintenance of intracellular redox status. In addition, Asc reduces $\alpha$-tocopheroxy radicals and tocopherone cations in the aqueous phase-membrane interface and contributes to the regeneration of tocopherol functioning by inhibiting ROS and free radicals within the cell membrane (23). Asc2P is a putative membrane-reinforcer that plays an immediate role in preventing membrane oxidative disruption through tocopherol recycling. In the present study, the possibility of UVB-protective effects, such as those exerted by titanium and zinc or the chestnut Castanea crenatat, was denied, since aspiration and rinsing were conducted before UVB irradiation so as to thoroughly remove extracellular Asc2P.

At the same UVB doses, less DNA damage was observed in keratinocytes than in fibroblasts. It has been reported that keratinocytes have higher DNA-repairing activity, but are also more susceptibile to apoptosis than fibroblasts (24). The accumulation of UV-induced DNA damage is one of the factors that causes carcinogenesis. It is therefore critical to defend keratinocytes against low intensity UVB damage that results in these cells evading apoptosis.

The tumor suppressor gene p53 undergoes quantitative and qualitative modulation by various biogenic stresses, and regulates genetic transcription of its downstream genes to arrest the cell cycle, leading cells to apoptosis. In the present study, we showed that p53 was expressed $1 \mathrm{~h}$ after UVB irradiation, whereas MDM2 underwent transcriptional control by p53 and was expressed 1 or $2 \mathrm{~h}$ after p53. In addition, the maximum expression of MDM2 occurred at $10 \mathrm{~mJ} / \mathrm{cm}^{2}$, whereas the maximum expression of p53 occurred at a higher irradiance level of $20-100 \mathrm{~mJ} / \mathrm{cm}^{2}$. The results suggest that the increase in the total expression of p53 at $20-100 \mathrm{~mJ} / \mathrm{cm}^{2}$ was attributed to a more marked increase in mt-p53 rather than wt-p53 expression. Thus, p53 may be a gene expressed so rapidly that it is capable of dealing with biogenic stress, including UV irradiation.

Mutation proteins occur when damage-accumulated DNA is replicated or transcribed immediately after UV irradiation. Prevention of this protein mutagenesis requires the alert and agile mobility of p53 expression. Thus, it is not surprising that p53 expression was observed as early as 1 or $2 \mathrm{~h}$ after UV irradiation. In addition, expression of the $\mathrm{mdm} 2$ gene seems to be induced by wt-p53, and MDM2 proteins bind p53 proteins and play modulating roles in the inhibition of the transcriptional ability of $\mathrm{p} 53$ and the decomposition of $\mathrm{p} 53$ proteins. The actual effective function of $\mathrm{p} 53$ proteins is considered to be restricted 
until 2 or $3 \mathrm{~h}$ after UV irradiation, and thereafter to be inactivated by MDM2 proteins, which are generated by induction attributed to p53 itself. In contrast, the expression of p53 was reduced by irradiation with a UV intensity range greater than $100 \mathrm{~mJ} / \mathrm{cm}^{2}$, corresponding to double or more the minimum erythema dose. p53 expression is markedly suppressed by UV irradiation at doses of $100-500 \mathrm{~mJ} / \mathrm{cm}^{2}$. This may occur because p53 genes suffer breakdown or extensive lesions at higher UV doses, and as a result cannot be expressed.

DNA lesions are accumulated rather than repaired in cells irradiated with UV rays at high doses, which makes cell cycle arrest impossible due to the failure of normal wt-p53 induction. Thus, intense UV radiation exposure may contribute to malignant cellular transformation regardless of the presence of p53. In addition, it was shown that, in cells irradiated with intense UV, the expression of mt-p53 increases; however, this corresponds to diminished expression of wt-p53. Mutated or variant-type p53 is commonly found in various types of malignant tumors, and is known to lead to the dominant-negative inhibition of wild-type p53 function. Using Western blot analysis (Figs. 5 and 6), the present study revealed that Asc2P, but not Asc, inhibited the UVB-induced expression of p53. According to immunocytochemical staining, this was composed mostly of mt-p53. It is possible that Asc2P exerts a preventive effect on carcinogenesis by suppressing the dominant-negative action exerted by mt-p53 on wt-p53 through the retention of a homeostatic redox state, rather than by suppressing the UVB-induced mutation of p53 itself. Furthermore, Asc2P may be responsible for the inhibition of intracellular ROS, the resulting mitigation of cellular oxidative stress, DNA damage suppression and the Asc-based promotion of diverse hydroxylation metabolisms, including collagen triple-strand-helix formation. Asc2P may also strongly suppress mt-p53 rather than wt-p53 expression.

In conclusion, the present study demonstrated that Asc2P inhibited UVB-induced harmful effects such as DNA damage, including CPD and 6-4PP damage, and the expression of mt-p53, concurrently with the suppression of marked wt-p53 expression. In addition, it maintained the normal cellular redox state and cell morphology.

\section{Acknowledgements}

The authors thank Dr Akira Ito of the Department of Dermatology, Kobe University School of Medicine for technical assistance. The present study was in part supported by grants-in-aid to N.M. from the Nagase Science and Technology Foundation and the Cosmetology Research Foundation

\section{References}

1. Garmyn M, Yaar M, Boileau N, Backendorf C and Gilchrest BA Effect of aging and habitual sun exposure on the genetic response of cultured human keratinocytes to solar-simulated irradiation. J Invest Dermatol 99: 743-748, 1992.

2. Kripke ML, Cox PA, Alas LG and Yarosh DB: Pyrimidine dimers in DNA initiate systemic immunosuppression in UV-irradiated mice. Proc Natl Acad Sci USA 89: 7516-7520, 1992.

3. Munson PJ, Hauser J, Levine AS and Dixon K: Test of models for the sequence specificity of UV-induced mutations in mammalian cells. Mutat Res 179: 103-114, 1987.

4. Matsunaga T, Hieda $\mathrm{K}$ and Nikaido $\mathrm{O}$ : Wavelength dependent formation of pirimidine dimers and (6-4) photoproducts in DNA by monochromatic ultraviolet light ranging from 150 to $365 \mathrm{~nm}$. Photochem Photobiol 54: 403-410, 1991.
5. Gilchrest BA, Garmyn M and Yaar M: Aging and photoaging affect gene expression in cultured human keratinocytes. Arch Dermatol 130: 82-86, 1994.

6. Sugimoto M, Yamashita R and Ueda M: Telomere length of the skin in association with chronological aging and photoaging. J Dermatol Sci 43: 43-47, 2006.

7. Hainaut P: The tumor suppressor protein p53: a receptor to genotoxic stress that controls cell growth and survival (review). Curr Opin Oncol 7: 76-82, 1995.

8. Inoue T, Geyer RK, Howard D, Yu ZK and Maki CG: MDM2 can promote the ubiquitination, nuclear export and degradation of $\mathrm{p} 53$ in the absence of direct binding. J Biol Chem 276: 45255-45260, 2001.

9. Gusterson BA, Anbazhagan R, Warren W, Midgely C, Lane DP, O'Hare M, Stamps A, Carter R and Jayatilake H: Expression of p53 in premalignant and malignant squamous epithelium. Oncogene 6: 1785-1789, 1991.

10. Kubo Y, Urano Y, Yoshimoto K, Iwahana H, Fukuhara K, Arase S and Itakura M: p53 gene mutations in human skin cancers and precancerous lesions: comparison with immunohistochemical analysis. J Invest Dermatol 102: 440-444, 1994.

11. Milner J and Medcalf EA: Cotranslation of activated mutant $\mathrm{p} 53$ with wild type drives the wild-type $\mathrm{p} 53$ protein into the mutant conformation. Cell 65: 765-774, 1991.

12. Strano S, Dell'Orso S, Di Agostino S, Fontemaggi G, Sacchi A and Blandino G: Mutant p53: an oncogenic transcription factor (review). Oncogene 26: 2212-2219, 2007.

13. Gonzalez S and Pathak MA: Inhibition of ultraviolet-induced formation of reactive oxygen species, lipid peroxidation, erythema and skin photosensitization by polypodium leucotomos. Photodermatol Photoimmunol Photomed 12: 45-56, 1996.

14. Runger TM, Epe B and Moller K: Processing of directly and indirectly ultraviolet-induced DNA damage in human cells. Recent Results Cancer Res 139: 31-42, 1995.

15. Housset B: Biochemical aspects of free radicals metabolism (review). Bull Eur Physiopathol Respir 23: 287-290, 1987.

16. Eguchi M, Miyazaki T, Masatsuji-Kato E, Tsuzuki T, Oribe T and Miwa N: Cytoprotection against ischemia-induced DNA cleavages and cell injuries in the rat liver by pro-vitamin $\mathrm{C}$ via hydrolytic conversion into ascorbate. Mol Cell Biochem 252: $17-23,2003$.

17. Furumoto K, Inoue E, Nagao N, Hiyama E and Miwa N: Agedependent telomere shortening is slowed down by enrichment of intracellular vitamin $\mathrm{C}$ via suppression of oxidative stress. Life Sci 63: 935-948, 1998.

18. Yokoo S, Furumoto K, Hiyama E and Miwa N: Slow-down of age-dependent telomere shortening is executed in human skin keratinocytes by hormesis-like-effects of trace hydrogen peroxide or by anti-oxidative effects of pro-vitamin $\mathrm{C}$ in common concurrently with reduction of intracellular oxidative stress. J Cell Biochem 93: 588-597, 2004.

19. Nagao N, Nakayama T, Etoh T, Saiki I and Miwa N: Tumor invasion is inhibited by phosphorylated ascorbate via enrichment of intracellular vitamin $\mathrm{C}$ and decreasing of oxidative stress. J Cancer Res Clin Oncol 126: 511-851, 2000.

20. Saitoh Y and Miwa N: Cytoprotection of vascular endotheliocytes by phosphorylated ascorbate through suppression of oxidative stress that is generated immediately after post-anoxic reoxygenation or with alkylhydroperoxides. J Cell Biochem 93: 653-663, 2004.

21. Sugimoto M, Okugawa Y and Miwa N: Preventive effects of phosphorylated ascorbate on ultraviolet-B induced apoptotic cell death and DNA strand cleavage through enrichment of intracellular vitamin $\mathrm{C}$ in skin epidermal keratinocytes. Free Radic Res 40: 213-221, 2006.

22. Shiu CT and Lee TM: Ultraviolet-B-induced oxidative stress and responses of the ascorbate-glutathione cycle in a marine macroalga Ulva fasciata. J Exp Bot 56: 2851-2865, 2005.

23. Kagan V, Witt E, Goldman R, Scita G and Packer L: Ultraviolet light-induced generation of vitamin $\mathrm{E}$ radicals and their recycling. A possible photosensitizing effect of vitamin E in skin. Free Radic Res Commun 16: 51-64, 1992.

24. D'Errico M, Lemma T, Calcagnile A, Proietti de Santis L and Dogliotti E: Cell type and DNA damage specific response of human skin cells to environmental agents. Mutat Res 614: 37-47, 2007. 